\title{
A UNIVERSAL EXHAUSTING DOMAIN
}

\author{
B. L. FRIDMAN
}

Abstract. A bounded domain $D \subset C^{n}$ is constructed such that every domain $G \subset \mathbf{C}^{n}$ is a monotone union of biholomorphic images of $D$.

I. Introduction. It is widely known that two domains in $\mathbf{C}^{n}, n>1$, are very rarely biholomorphically equivalent. In this paper we construct a domain $D \subset \mathrm{C}^{n}, n \geqslant 1$, that can be used to approximate any domain in $\mathbf{C}^{n}$ and therefore is "almost equivalent" to any domain in $\mathbf{C}^{n}$.

Let $D, G$ be domains in $\mathbf{C}^{n}$. We will say that $G$ can be exhausted by $D$ if for every compact $K \subset G$ there exists a biholomorphic imbedding $F: D \rightarrow G$ such that $F(D) \supset K$.

Given $D$ the question is to describe such domains $G$ that can be exhausted by $D$. Related questions are discussed in [1-5]. If $G$ is a complete hyperbolic manifold, the following two results are known. If $D$ is a ball, polydisk or any bounded homogeneous domain, then there is only one choice of $G$, that is $G$ is biholomorphically equivalent to $D$ (see $[2,4])$. If $D$ is strictly pseudoconvex with a $C^{3}$ boundary, then $G$ is biholomorphically equivalent to either $D$ or to $B$, the unit ball in $C^{n}$ (see [4]).

In this paper we are going to construct a universal exhausting domain.

THEOREM 1. There exists a bounded domain $D \subset \mathbf{C}^{n}, n \geqslant 1$, such that every domain $G \subset \mathbf{C}^{n}$ can be exhausted by $D$.

Corollary 1. There exists a bounded domain $D \subset \mathbf{C}^{n}$ such that every domain $G \subset \mathbf{C}^{n}$ is a monotone union of biholomorphic images of $D$.

This means that $G=\bigcup_{s=1}^{\infty} F_{s}(D)$, where $F_{s}: D \rightarrow G$ is a biholomorphic imbedding and $F_{s}(D) \subset F_{s+1}(D)$ for all $s$.

The construction of a universal domain allows us also to prove

THEOREM 2. There exist two bounded domains $D_{1}, D_{2}$ in $\mathbf{C}^{n}$ such that each of them can be exhausted by the other but they are not biholomorphically equivalent.

II. Construction of a universal exhausting domain. We use the following notations. If $z \in \mathbf{C}^{n}, n>1$, then $z=\left(z_{1}, z^{\prime}\right)$, where $z^{\prime}=\left(z_{2}, \ldots, z_{n}\right) . B(z, r)=\{w \in \mathbf{C} \mid$ $|w-z|<r\} ; B=B(0,1)$, the unit ball in $C^{n}$. $\partial D$ is the boundary of $D . p, q$ are points on $\partial B, p=(1,0, \ldots, 0), q=-p$.

Received by the editors September 24, 1985.

1980 Mathematics Subject Classification (1985 Revision). Primary 32H99. 
$\operatorname{Aut}(B)$ is the group of holomorphic automorphisms of $B$. Information about the structure and properties of $\operatorname{Aut}(B)$ can be found in [6].

1. LEMMA 1. For any $\varepsilon>0$ and $R>0$ there exist $r>0$ and $T \in \operatorname{Aut}(B)$ such that (1.1) $R>r>0$,

(1.2) $T(B \backslash B(p, R)) \subset B(q, \varepsilon)$,

(1.3) $T(\overline{B(p, r)} \cap B) \subset B(p, \varepsilon)$.

Proof. Consider for $0<\lambda<1, T_{\lambda} \in \operatorname{Aut}(B)$,

$$
T_{\lambda} z=\left\{\frac{z_{1}-\lambda}{1-z_{1} \lambda}, \sqrt{1-\lambda^{2}} \frac{z^{\prime}}{1-z_{1} \lambda}\right\} .
$$

One can see that for $z=\left(z_{1}, z^{\prime}\right) \in V=B \backslash B(p, R), \operatorname{Re} z_{1}<1-\nu$, where $\nu>0$. Therefore, when $\lambda \rightarrow 1, T_{\lambda}(V) \rightarrow q$ uniformly on $V$. So, for given $\varepsilon>0$ we can find $\lambda_{0}, T=T_{\lambda_{0}}$ such that (1.2) is satisfied. (1.1) and (1.3) can now be satisfied by choosing a small enough $r>0$. It is possible because $T(p)=p$ and $T$ is continuous at $p$.

2. LEMMA 2. For any domain $U$ which is a finite union of open balls, $U=$ $\bigcup_{s=1}^{N} B\left(z^{s}, r_{s}\right)$, and any compact $K \subset U$, there exist an $\varepsilon>0$ and $F: U \rightarrow C^{n}, F$ is holomorphic, such that

(2.1) $F(U) \cap B$ is connected,

(2.2) $W(\varepsilon) \supset F(K)$, where $W(\varepsilon)=B \backslash(B(p, \varepsilon) \cup B(q, \varepsilon))$,

(2.3) $F(U) \supset B(p, \varepsilon) \cup B(q, \varepsilon)$,

(2.4) $F^{-1}$ is one-to-one on $F(U) \cap B$.

Proof. (1) First we take a ball of a minimal radius that contains $U$. Without any loss of generality we can assume that this ball is the unit ball $B$. One can prove now that $\partial B \cap \partial U$ contains at least two different points $\zeta, \eta$.

(2) Now we find a $T \in \operatorname{Aut}(B)$ such that $T \zeta=p, T \eta=q . T$ is analytic in a neighborhood $B_{0}$ of $\bar{B}$.

(3) We find now such a small $\delta>0$ that if we introduce $\phi_{\delta}: \mathbf{C}^{n} \rightarrow \mathbf{C}^{n} ; \phi_{\delta}$ : $z \mapsto(1+\delta) z$, then $\phi_{\delta}(U)$ has the following properties:

(a) $\phi_{\delta}(U) \subset B_{0}$,

(b) $\phi_{\delta}(U) \cap B$ is connected,

(c) $\phi_{\delta}(U) \cap B \supset \phi_{\delta}(K)$.

(4) We take now $F=T \circ \phi_{\delta}$.

(2.1) and (2.4) follow from the construction of $F$. The existence of an $\varepsilon>0$ such that (2.2) and (2.3) are satisfied follows from the facts that $\phi_{\delta}(U) \ni \zeta, \eta$, and therefore $F(U) \ni p, q$, that $F(K) \subset B$, and that $F(U)$ is open.

3. Let as before a domain $U=\bigcup_{i=1}^{N} B\left(z^{i}, r_{i}\right)$.

We take

$$
K_{s}=\bigcup_{i=1}^{N} \overline{B\left(z^{i}, r_{i}-1 / s\right)} .
$$

$\left\{K_{s}\right\}$ is a sequence of compacts in $U$ such that

(a) $K_{s} \subset K_{s+1}$ for every $s \geqslant 1$.

(b) $\cup_{s=1}^{\infty} K_{s}=U$. 
(c) If $K$ is any compact in $U$, then there exists an $s$ such that $K \subset K_{s}$.

Let $1>R>r>0$. Denote, for $\zeta \in \partial B$,

$$
D(R, r, \zeta)=[B(\zeta, r) \backslash \overline{B(\zeta, r)}] \cap B .
$$

$0<R<1$ and a point $\zeta \in \partial B$ are now given. $U=\bigcup_{s=1}^{\infty} K_{s}$ is from above.

LEMmA 3. There exist sequences $\left\{R_{s}\right\}$ and $\left\{r_{s}\right\}$ and closed sets $V_{s}, 1 \leqslant s<\infty$, such that, for all $s \geqslant 1$,

(3.1) $R_{1}=R$,

(3.2) $R_{s}>r_{s}>R_{s+1}>0$,

(3.3) $V_{s} \subset D_{s}$, where $D_{s}=D\left(R_{s}, r_{s}, \zeta\right)$.

(3.4) There exists a biholomorphic imbedding $\phi_{s}:\left(B \backslash V_{s}\right) \rightarrow U$ such that

$$
\phi_{s}\left(D_{s} \backslash V_{s}\right) \supset K_{s} \text {. }
$$

(3.5) $B \backslash V_{s}$ is connected.

Proof. Using a unitary transformation (if needed), we may assume $\zeta=p$.

We will construct $R_{s}$ by induction. For every $R_{s}$ we construct $r_{s}, V_{s}, \phi_{s}$ and then $R_{s+1}$.

For $s=1$ we take $R_{1}=R$. Suppose $R_{s}$ has been constructed. Using Lemma 2 we can find $F=F_{s}: U \rightarrow C^{n}$ such that (2.1)-(2.4) hold for some $\varepsilon=\varepsilon_{s}>0$ and $K=K_{s}$. Applying Lemma 1 now for $\varepsilon_{s}$ and $R_{s}$ we find $r=r_{s}>0$ and $T=T_{s} \in$ $\operatorname{Aut}(B)$ such that (1.1)-(1.3) hold. From (1.2), (1.3) and (2.2) we find

$$
T_{s}\left(D_{s}\right) \supset W\left(\varepsilon_{s}\right) \supset F_{s}\left(K_{s}\right) .
$$

We choose now $V_{s}=T_{s}^{-1}\left(\overline{W\left(\varepsilon_{s}\right)} \backslash F_{s}(U)\right), \phi_{s}=F_{s}^{-1} \circ T_{s}$ and $R_{s+1}$ is any positive number less than $r_{s}$.

Properties (3.1)-(3.5) can be checked now by using (2.1)-(2.4).

4. Consider the set $S$ of all such domains $U$ each of which is a finite union of open balls in $\mathbf{C}^{n}$ with centers at rational points and rational radii. Evidently, $S$ is countable. $S=\left\{U_{1}, U_{2}, \ldots, U_{m}, \ldots\right\}$

5. We set, for $m \geqslant 1, \zeta_{m}=(\exp (\pi i / m), 0, \ldots, 0) \in \partial B$. Choose now numbers $R\left(\zeta_{m}\right), m \geqslant 1$, such that $R\left(\zeta_{m}\right)>0$ and $B\left(\zeta_{m}, R\left(\zeta_{m}\right)\right) \cap B\left(\zeta_{s}, R\left(\zeta_{s}\right)\right)=\varnothing$ if $m \neq s$. One can take, say, $R\left(\zeta_{m}\right)=\left|\zeta_{m+1}-\zeta_{m}\right| / 2$. For each $m$ we use now the paragraph 3 to represent $U_{m}=\bigcup_{s=1}^{\infty} K_{m s}$ and then Lemma 3 (where $\zeta=\zeta_{m}, R=R\left(\zeta_{m}\right)$ ) to find $V_{m s}$ and $\phi_{m s}$. Let

$$
D=B \backslash \bigcup_{m, s=1}^{\infty} V_{m s} .
$$

Connectedness of $D$ follows from the construction of all $V_{m s}$ (each $V_{m s}$ lies in a different open set) and (3.5). The universal property of $D$ follows from the following. Given a domain $G \subset \mathbf{C}^{n}$ and a compact $K \subset G$ we can always find $m, s$ such that $U_{m} \in S, G \supset U_{m} \supset K_{m s} \supset K$. From the construction and (3.4) one can conclude now that $\phi_{m s}: D \rightarrow U_{m}$ is a biholomorphic imbedding and $\phi_{m s}(D) \supset K_{m s}$. So, $G \supset \phi_{m s}(D) \supset K$. 
III. 1. Proof of Theorem 1 follows from the construction of $D$ and was presented above.

2. Proof of the Corollary 1. One can always represent a domain $G$ as $G=\bigcup_{s=1}^{\infty} G_{s}$ such that, for all $s \geqslant 1$,

(a) $G_{s}$ is a subdomain in $G$,

(b) $\bar{G}_{s}$ is a compact set in $G$,

(c) $G_{s+1} \supset \bar{G}_{s}$.

Now using Theorem 1 take $F_{s}: D \rightarrow G_{s+1}$ such that $F_{s}$ is a biholomorphic imbedding and $F_{s}(D) \supset \bar{G}_{s}$. Eviently, $F_{s+1}(D) \supset F_{s}(D)$ and $G=\cup_{s=1}^{\infty} F_{s}(D)$.

3. Proof of Theorem 2. $V_{s}$ was constructed in Lemma 3. One can see that we can require in addition to (3.1)-(3.5) the following: $V_{s}$ has no isolated points. Actually if we take $V_{s}$ constructed before and add sufficiently small neighborhoods of its isolated points the closure of the new set will still satisfy (3.3)-(3.5). Now, using this we see that a domain $D_{1}=D$ can be constructed in such a way that its boundary does not have any isolated points, but $D_{1}$ still satisfies Theorem 1 . Let $a \in D_{1}$, choose $D_{2}=D_{1} \backslash\{a\}$. $D_{2}$ will also be a universal exhausting domain.

Since $D_{1}$ and $D_{2}$ both are universal exhausting domains they are mutually exhaustable.

Now we need to prove that $D_{2}$ is not holomorphically equivalent to $D_{1}$. If it is, then let $F: D_{2} \rightarrow D_{1}$ be a biholomorphism. $F$ has a removable singularity at $a \in \partial D_{2}$. So, $F$ can be uniquely extended to $\{a\}$ as a holomorphic map. Let $F(a)=b \in \bar{D}_{1} . b \in D_{1}$ since $F$ is an open map and $\partial D_{1}$ has no isolated singularities. Let $c \in D_{2}$ be such a point that $F(c)=b$. Now if $W_{c} \cap W_{a}=\varnothing$ are neighborhoods of $c$ and $a$, respectively, $F\left(W_{c}\right) \cap F\left(W_{a}\right)$ is not empty and open. This contradicts the suggestion that $F$ is one-to-one on $D_{2}$.

\section{REFERENCES}

1. H. Alexander, Extremal holomorphic imbeddings between ball and polydisc, Proc. Amer. Math. Soc. 68 (1978), 200-202.

2. J.-E. Fornaess and E. L. Stout, Polydiscs in complex manifolds, Math. Ann. 227 (1977), 145-153.

3. J.-E. Fornaess and Sibony Nessim, Increasing sequences of complex manifolds, Math. Ann. 255 (1981), 351-360.

4. B. L. Fridman, Biholomorphic invariants of a hyperbolic manifold and some applications, Trans. Amer. Math. Soc. 276 (1983), 685-698.

5. L. Lempert, $A$ note on mapping polydiscs into balls and vice versa, Acta Math. Hungar. 34 (1979), 117-119.

6. W. Rudin, Function theory in the unit ball of $\mathbf{C}^{n}$, Springer-Verlag, New York, 1980.

Department of Mathematics, Wichita State University, Wichita, Kansas 67208 